\title{
Finite-Time Stability and Stabilization of Networked Control Systems with Bounded Markovian Packet Dropout
}

\author{
Yeguo Sun and Guanjun Li \\ Department of Mathematics and Computational Science, Huainan Normal University, \\ 238 Dongshan West Road, Huainan 232038, China
}

Correspondence should be addressed to Yeguo Sun; ygsun@hnnu.edu.cn

Received 7 January 2014; Accepted 25 February 2014; Published 23 March 2014

Academic Editor: Jinde Cao

Copyright (C) 2014 Y. Sun and G. Li. This is an open access article distributed under the Creative Commons Attribution License, which permits unrestricted use, distribution, and reproduction in any medium, provided the original work is properly cited.

The finite-time stability and stabilization problems of a class of networked control systems (NCSs) with bounded Markovian packet dropout are investigated. The main results provided in the paper are sufficient conditions for finite-time stability and stabilization via state feedback. An iterative approach is proposed to model NCSs with bounded packet dropout as jump linear systems (JLSs). Based on Lyapunov stability theory and JLSs theory, the sufficient conditions for finite-time stability and stabilization of the underlying systems are derived via linear matrix inequalities (LMIs) formulation. Lastly, an illustrative example is given to demonstrate the effectiveness of the proposed results.

\section{Introduction}

The concept of Lyapunov asymptotic stability is largely known to the control community; see [1] and the references therein. However, often Lyapunov asymptotic stability is not enough for practical applications, because there are some cases where large values of state variables are not acceptable. For this purpose, the concept of finite-time stability (FTS) can be used. Some early results on FTS can be found in [2]; more recently the concept of FTS has been revisited in the light of recent results coming from linear matrix inequalities (LMIs) theory, which has made it possible to find less conservative conditions for guaranteeing FTS and finite time stabilization of discrete-time and continuous-time systems [3, 4]. In [5], finite-time stabilization of linear time-varying systems has been studied. In [6-8], finite-time control problem for the impulsive systems is discussed. In [9], sufficient conditions for finite-time stability and stabilization of a class of nonlinear quadratic systems are also presented. For more analysis and synthesis results of finite control problem, the readers are referred to the literature $[10,11]$ and the references therein. In [12], sufficient conditions for finite-time boundness and stability of switched system are proposed, and static state and dynamic output feedback controllers are designed to finitetime stabilise switched linear systems. In [13], a new design approach is proposed for robust finite-time $H_{\infty}$ control of a class of Markov jump systems with partially known information. In [14], finite-time reliable guaranteed cost fuzzy control for discrete-time nonlinear systems with actuator faults is investigated. In [15], the finite-time stochastic synchronization problem for complex networks with stochastic noise perturbations is studied. In [16], finite-time synchronization of the singular hybrid coupled networks is investigated.

On the other hand, NCSs are feedback control systems with control loops closed via digital communication channels. Compared with the traditional point-to-point wiring, the use of the communication channels can reduce the costs of cables and power, simplify the installation and maintenance of the whole system, and increase the reliability. Because of these attractive benefits, many industrial companies and institutes have shown interest in applying networks for remote industrial control purposes and factory automation [17]. However, the insertion of communication networks in feedback control loops makes the NCSs analysis and synthesis complex; see [18-20] and the references therein, where much attention has been paid to the delayed data packets of an NCSs due to network transmissions. In fact, data packets through networks suffer not only transmission delays, but also, possibly, packet dropout [21, 22]; the latter is a potential source of instability and poor performance in 


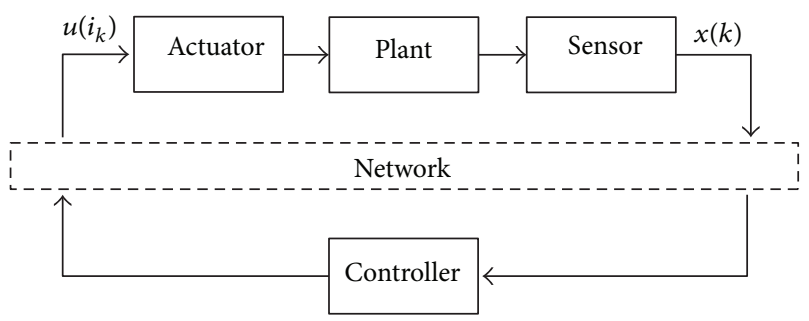

FIGURE 1: Illustration of NCSs over communication network.

NCSs because of the critical real-time requirement in control systems.

So far, Lyapunov stability analysis for NCSs with packet dropout has been extensively studied by many researchers [23-25]. However, to date and to the best of our knowledge, the problems of finite-time stabilization of NCSs with Markovian packet dropout have not been fully investigated and still remain challenging, although results related to systems over networks with packet loss are reported in the existing literature, which motivates the study of this paper. The main contributions are given as follows: (1) definitions of finitetime stabilization are extended to NCSs with packet dropout; (2) sufficient conditions for finite-time stabilization in terms of linear matrix inequalities formulation are given.

This paper is organized as follows. An iterative method to model NCSs with bounded Markovian packet dropout as MJLSs is proposed in Section 2. The stochastic stability and stabilization conditions for NCSs are derived via LMIs in Section 3. Section 4 provides numerical examples to illustrate the effectiveness of our results. Finally, Section 5 gives some concluding remarks.

Notations. Throughout this paper, for two matrices $A$ and $B$, $A<B$ means that $A-B$ is negative definite, and $A^{T}$ denotes the transpose of $A . E\{\cdot\}$ stands for the mathematical expectation operator. $\lambda_{\max }(A)$ and $\lambda_{\text {min }}(A)$ denote the maximum and minimum eigenvalue of $A$, respectively. $I$ is the identity matrix.

\section{Problem Formulation and Preliminaries}

The framework of NCSs considered in this paper is depicted in Figure 1, and the plant to be controlled is described by the following linear discrete-time systems:

$$
x(k+1)=A x(k)+B u(k),
$$

where $x(k) \in \mathbb{R}^{n}$ is the state and $u(k) \in \mathbb{R}^{m}$ is the control input. $A$ and $B$ are known real constant matrices with appropriate dimensions. We assume that $(A, B)$ is controllable.

Let $\mathscr{I}=\left\{i_{1}, i_{2}, \ldots\right\}$, which is a subsequence of $\mathbb{N}=$ $\{1,2, \ldots\}$, denote the sequence of time points of successful date transmission from sensor to actuator. The state feedback controller law is

$$
u(k)=K x(k),
$$

where $K \in \mathbb{R}^{m \times n}$ is to be designed. The control input is held at the previous value by the zero-order hold during the two successively successful transmitted instant; that is

$$
u(l)=u\left(i_{k}\right)=K x\left(i_{k}\right), \quad i_{k} \leq l \leq i_{k+1}-1 .
$$

Thus the closed-loop system is

$$
x(l+1)=A x(l)+B K x\left(i_{k}\right), \quad i_{k} \leq l \leq i_{k+1}-1 .
$$

Applying iteratively (4), we can obtain

$$
x\left(i_{k+1}\right)=\left(A^{i_{k+1}-i_{k}}+\sum_{r=0}^{i_{k+1}-i_{k}-1} A^{r} B K\right) x\left(i_{k}\right), \quad i_{k} \in \mathscr{I} .
$$

Define the packet dropout process as follows:

$$
r\left(i_{k}\right)=i_{k+1}-i_{k},
$$

which takes values in the finite state space $\mathcal{S}=\{1,2, \ldots, s\}$, where $s$ is defined as

$$
s=\max _{i_{k} \in \mathscr{I}}\left(i_{k+1}-i_{k}\right) .
$$

Then the closed-loop system (5) can be rewritten as jump linear systems:

$$
x\left(i_{k+1}\right)=\left(A^{r\left(i_{k}\right)}+\sum_{r=0}^{r\left(i_{k}\right)-1} A^{r} B K\right) x\left(i_{k}\right), \quad i_{k} \in \mathscr{I} .
$$

The packet dropout process $\left\{r\left(i_{k}\right)\right\}$ is described by a discretetime homogeneous Markov chain with mode transition probabilities:

$$
\pi_{i j}=\mathbb{P}\left(r\left(i_{k+1}\right)=j \mid r\left(i_{k}\right)=i\right) \geq 0, \quad \forall i, j \in \mathcal{S} .
$$

The corresponding transition probabilities is defined as

$$
\Pi=\left[\begin{array}{cccc}
\pi_{11} & \pi_{12} & \cdots & \pi_{1 s} \\
\pi_{21} & \pi_{22} & \cdots & \pi_{2 s} \\
\vdots & \vdots & \ddots & \vdots \\
\pi_{s 1} & \pi_{s 2} & \cdots & \pi_{s s}
\end{array}\right] .
$$

Remark 1. It is worth pointing out that the packet dropout process $\left\{r\left(i_{k}\right), i_{k} \geq 0\right\}$ includes both sensor-to-controller and controller-to-actuator packet dropouts.

The main aim of this paper is to find some sufficient conditions which guarantee that the system given (8) is stable over a finite-time interval. This concept can be formalized through the following definition.

Definition 2. System (8) is said to be finite-time stable with respect to $(\alpha, \beta, R, N)$, where $R$ is a positive-definite matrix, $0<\alpha<\beta$, if

$$
\begin{array}{r}
x^{T}\left(i_{0}\right) R x\left(i_{0}\right) \leq \alpha^{2} \Longrightarrow E\left\{x^{T}\left(i_{k}\right) R x\left(i_{k}\right)\right\} \leq \beta^{2}, \\
k \in\{1, \ldots, N\} .
\end{array}
$$


To this end, the following lemmas will be essential for the proofs in the next section and theirs proofs can be found in the cited reference.

Lemma 3 (see [26]). For any matrices $U \in R^{n \times n}$ and $V \in$ $R^{n \times n}$, if the matrix $V>0$, then one has

$$
U^{T}+U-V \leq U^{T} V^{-1} U .
$$

Lemma 4 (Schur complement lemma; see [26]). For a given symmetric matrix

$$
W=\left[\begin{array}{ll}
W_{11} & W_{12} \\
W_{12}^{T} & W_{22}
\end{array}\right],
$$

where $W_{11} \in \mathbb{R}^{p \times p}, W_{22} \in \mathbb{R}^{q \times q}$, and $W_{12} \in \mathbb{R}^{p \times q}$, then the following three conditions are mutually equivalent:

(1) $W<0$,

(2) $W_{11}<0, W_{22}-W_{12}^{T} W_{11}^{-1} W_{12}<0$,

(3) $W_{22}<0, W_{11}-W_{12} W_{22}^{-1} W_{12}^{T}<0$.

\section{Main Results}

In this section, we will find a state feedback control matrix $K$, such that system (8) is finite-time stable with respect to $(\alpha, \beta, R, N)$. In order to solve the problem, the following theorem will be essential.

Theorem 5. For a constant $\gamma>0$, system (8) is finite-time stable with respect to $(\alpha, \beta, R, N)$ if there exist matrix $P_{i}>0$, $i \in \mathcal{S}$, and two scalars $\lambda_{1}, \lambda_{2}$, such that the following conditions hold:

$$
\begin{gathered}
\lambda_{1} I \leq P_{i} \leq \lambda_{2} I, \\
(\gamma+1)^{N} \alpha^{2} \lambda_{2}-\beta^{2} \lambda_{1}<0, \\
\sum_{j=1}^{s} \pi_{i j}\left(A^{j}+B_{j} K\right)^{T} \widetilde{P}_{j}\left(A^{j}+B_{j} K\right)-(\gamma+1) \widetilde{P}_{i}<0,
\end{gathered}
$$

where

$$
\widetilde{P}_{i}=R^{1 / 2} P_{i} R^{1 / 2}, \quad B_{j}=\sum_{r=0}^{j-1} A^{r} B .
$$

Proof. Choose the Lyapunov function as

$$
V\left(x\left(i_{k}\right), i\right)=x^{T}\left(i_{k}\right) \widetilde{P}_{i} x\left(i_{k}\right) .
$$

Firstly, we will prove that conditions (14) combined with the following condition

$$
\zeta V\left(x\left(i_{k}\right), r\left(i_{k}\right)\right)<\gamma V\left(x\left(i_{k}\right), r\left(i_{k}\right)\right)
$$

imply that (8) is finite-time stable with respect to $(\alpha, \beta, R, N)$, where $\zeta$ is difference operator and defined as follows:

$$
\begin{aligned}
\zeta V & \left(x\left(i_{k}\right), r\left(i_{k}\right)\right) \\
& =E\left\{V\left(x\left(i_{k+1}\right), r\left(i_{k+1}\right)\right)\right\}-V\left(x\left(i_{k}\right), r\left(i_{k}\right)\right) .
\end{aligned}
$$

Applying iteratively (18), we obtain

$$
\begin{aligned}
E\left\{V\left(x\left(i_{k}\right), r\left(i_{k}\right)\right)\right\} & <(\gamma+1) V\left(x\left(i_{k-1}\right), r\left(i_{k-1}\right)\right) \\
& <(\gamma+1)^{2} V\left(x\left(i_{k-2}\right), r\left(i_{k-2}\right)\right) \\
& \cdots \\
& <(\gamma+1)^{k} V\left(x\left(i_{0}\right), r\left(i_{0}\right)\right) .
\end{aligned}
$$

Note that

$$
\begin{aligned}
E\left\{V\left(x\left(i_{k}\right), r\left(i_{k}\right)\right)\right\} & =E\left\{x^{T}\left(i_{k}\right) \widetilde{P}_{r\left(i_{k}\right)} x\left(i_{k}\right)\right\} \\
& =E\left\{x^{T}\left(i_{k}\right) R^{1 / 2} P_{r\left(i_{k}\right)} R^{1 / 2} x\left(i_{k}\right)\right\} \\
& \geq \lambda_{\min }\left(P_{r\left(i_{k}\right)}\right) E\left\{x^{T}\left(i_{k}\right) R x\left(i_{k}\right)\right\}, \\
(\gamma+1)^{k} V\left(x\left(i_{0}\right), r\left(i_{0}\right)\right) & =(\gamma+1)^{k} x^{T}\left(i_{0}\right) \widetilde{P}_{r\left(i_{0}\right)} x\left(i_{0}\right) \\
& =(\gamma+1)^{k} x^{T}\left(i_{0}\right) R^{1 / 2} P_{r\left(i_{0}\right)} R^{1 / 2} x\left(i_{0}\right) \\
& \leq(\gamma+1)^{k} \lambda_{\max }\left(P_{r\left(i_{0}\right)}\right) x^{T}\left(i_{0}\right) R x\left(i_{0}\right) .
\end{aligned}
$$

Thus, we have

$$
\begin{aligned}
\lambda_{\min } & \left(P_{r\left(i_{k}\right)}\right) E\left\{x^{T}\left(i_{k}\right) R x\left(i_{k}\right)\right\} \\
& \leq(\gamma+1)^{k} \lambda_{\max }\left(P_{r\left(i_{0}\right)}\right) x^{T}\left(i_{0}\right) R x\left(i_{0}\right) \\
& \leq(\gamma+1)^{k} \lambda_{\max }\left(P_{r\left(i_{0}\right)}\right) \alpha^{2} .
\end{aligned}
$$

From conditions (14), we can obtain

$$
E\left\{x^{T}\left(i_{k}\right) R x\left(i_{k}\right)\right\} \leq(\gamma+1)^{k} \frac{\lambda_{\text {max }}\left(P_{r\left(i_{0}\right)}\right)}{\lambda_{\text {min }}\left(P_{r\left(i_{k}\right)}\right)} \alpha^{2} \leq \beta^{2} .
$$

Secondly, we will prove that condition (15) is equivalent to condition (18). According to the definition of difference operator $\zeta$ and the transition probabilities of the packet dropout process $\left\{r\left(i_{k}\right)\right\}$, we can obtain

$$
\begin{aligned}
\zeta V\left(x\left(i_{k}\right), r\left(i_{k}\right)\right) \\
\quad=E\left\{V\left(x\left(i_{k+1}\right), j\right) \mid x\left(i_{k}\right), i\right\}-V\left(x\left(i_{k}\right), i\right) \\
\quad=x^{T}\left(i_{k}\right)\left(\sum_{j=1}^{s} \pi_{i j}\left(A^{j}+B_{j} K\right)^{T} \widetilde{P}_{j}\left(A^{j}+B_{j} K\right)\right) x\left(i_{k}\right) \\
\quad \leq \gamma x^{T}\left(i_{k}\right) \widetilde{P}_{i} x\left(i_{k}\right),
\end{aligned}
$$

which yields that

$$
x^{T}\left(i_{k}\right)\left(\sum_{j=1}^{s} \pi_{i j}\left(A^{j}+B_{j} K\right)^{T} \widetilde{P}_{j}\left(A^{j}+B_{j} K\right)-(\gamma+1) \widetilde{P}_{i}\right) x\left(i_{k}\right)<0 .
$$


Now we turn back to our original problem, which is to find sufficient conditions which guarantee that the system (8) with the controller (2) is finite-time stable with respect to $(\alpha, \beta, R, N)$. The solution of this problem is given by the following theorem.

Theorem 6. For a constant $\gamma>0$, system (8) is finite-time stable with respect to $(\alpha, \beta, R, N)$ if there exist matrix $\widetilde{X}_{i}, i \in \mathcal{S}$, $G \in \mathbb{R}^{n \times n}, Y \in \mathbb{R}^{m \times n}$, and two scalars $\lambda_{1}, \lambda_{2}$, such that the following conditions hold:

$$
\begin{gathered}
\lambda_{2}^{-1} R^{-1} \leq \widetilde{X}_{i} \leq \lambda_{1}^{-1} R^{-1}, \\
\lambda_{1}^{-1}(\gamma+1)^{N} \alpha^{2}-\lambda_{2}^{-1} \beta^{2}<0, \\
{\left[\begin{array}{cc}
-G-G^{T}+(\gamma+1)^{-1} \widetilde{X}_{i} & M_{i} \\
M_{i}^{T} & -\Lambda
\end{array}\right]<0,}
\end{gathered}
$$

where

$$
\begin{gathered}
M_{i}=\left[\sqrt{\pi_{i 1}}\left(A G+B_{1} Y\right)^{T},\right. \\
\left.\sqrt{\pi_{i 2}}\left(A^{2} G+B_{2} Y\right)^{T}, \ldots, \sqrt{\pi_{i s}}\left(A^{s} G+B_{s} Y\right)^{T}\right], \\
\Lambda=\left[\begin{array}{cccc}
\widetilde{X}_{1} & 0 & 0 & 0 \\
0 & \widetilde{X}_{2} & 0 & 0 \\
0 & 0 & \ddots & 0 \\
0 & 0 & 0 & \widetilde{X}_{s}
\end{array}\right] .
\end{gathered}
$$

Then the desired stabilizable control matrix is given by

$$
K=Y G^{-1} \text {. }
$$

Proof. By Lemma 4, (27) is equivalent to

$$
\begin{aligned}
& \sum_{j=1}^{s} \pi_{i j}\left(A^{j} G+B_{j} Y\right)^{T} \widetilde{X}_{j}^{-1}\left(A^{j} G+B_{j} Y\right)-G-G^{T} \\
& +(\gamma+1)^{-1} \widetilde{X}_{i}<0 .
\end{aligned}
$$

From Lemma 3, we can obtain

$$
-G-G^{T}+(\gamma+1)^{-1} \widetilde{X}_{i} \geq-(\gamma+1) G^{T} \widetilde{X}_{i}^{-1} G
$$

Denote $\widetilde{P}_{i}=\widetilde{X}_{i}^{-1}$. From (30) and (31), we have

$$
\sum_{j=1}^{s} \pi_{i j}\left(A^{j} G+B_{j} Y\right)^{T} \widetilde{P}_{j}\left(A^{j} G+B_{j} Y\right)-(\gamma+1) G^{T} \widetilde{P}_{i} G<0 .
$$

Let $Y=K G$. Then, we can obtain

$$
\sum_{j=1}^{s} \pi_{i j}\left(A^{j} G+B_{j} K G\right)^{T} \widetilde{P}_{j}\left(A^{j} G+B_{j} K G\right)-(\gamma+1) G^{T} \widetilde{P}_{i} G<0,
$$

which yields that

$$
G^{T}\left(\sum_{j=1}^{s} \pi_{i j}\left(A^{j}+B_{j} K\right)^{T} \widetilde{P}_{j}\left(A^{j}+B_{j} K\right)-(\gamma+1) \widetilde{P}_{i}\right) G<0,
$$

which is equivalent to

$$
\sum_{j=1}^{s} \pi_{i j}\left(A^{j}+B_{j} K\right)^{T} \widetilde{P}_{j}\left(A^{j}+B_{j} K\right)-(\gamma+1) \widetilde{P}_{i}<0 .
$$

In view of Theorem 5, system (8) is finite-time stable with respect to $(\alpha, \beta, R, N)$. Moreover, the desired controller gain is given by (29).

Remark 7. For the case of $i_{k}<l \leq i_{k+1}-1$, the system transient performance can also be accommodated. In fact, denote $h\left(i_{k}\right)=l-i_{k} \in \mathcal{S}$. Then we have

$$
x(l)=\left(A^{h\left(i_{k}\right)}+\sum_{r=0}^{h\left(i_{k}\right)-1} A^{r} B K\right) x\left(i_{k}\right), \quad i_{k} \in \mathscr{I} .
$$

By Theorem 6, the system transient performance can be accommodated.

\section{Numerical Example}

In this section, a numerical example is given to illustrate the effectiveness of the proposed methods. Let us consider the continuous time system [27]:

$$
\dot{x}(t)=\bar{A} x(t)+\bar{B} u(t),
$$

where

$$
\bar{A}=\left[\begin{array}{ccc}
-1 & 0 & -0.5 \\
1 & -0.5 & 0 \\
0 & 0 & 0.5
\end{array}\right], \quad \bar{B}=\left[\begin{array}{l}
0 \\
0 \\
1
\end{array}\right]
$$

When the plant is sampled with a sampling period $T=0.5 \mathrm{~s}$, the corresponding discretized system is

$$
\begin{aligned}
x & (k+1) \\
& =\left[\begin{array}{ccc}
0.6065 & 0 & -0.2258 \\
0.3445 & 0.7788 & -0.0536 \\
0 & 0 & 1.2840
\end{array}\right] x(k)+\left[\begin{array}{c}
-0.0582 \\
-0.0093 \\
0.5681
\end{array}\right] u(k) .
\end{aligned}
$$

Both the continuous time system and discretized system are unstable because the eigenvalues of $\bar{A}$ are $-0.5,-1,0.5$ and the eigenvalues of $A$ are $0.7788,0.6065,1.2840$. Furthermore, we assume that the packet dropout upper bound is $s=4$ and the transition probabilities matrix is as follows:

$$
\Pi=\left[\begin{array}{llll}
0.3 & 0.2 & 0.1 & 0.4 \\
0.3 & 0.2 & 0.3 & 0.2 \\
0.5 & 0.1 & 0.1 & 0.3 \\
0.2 & 0.3 & 0.4 & 0.1
\end{array}\right]
$$




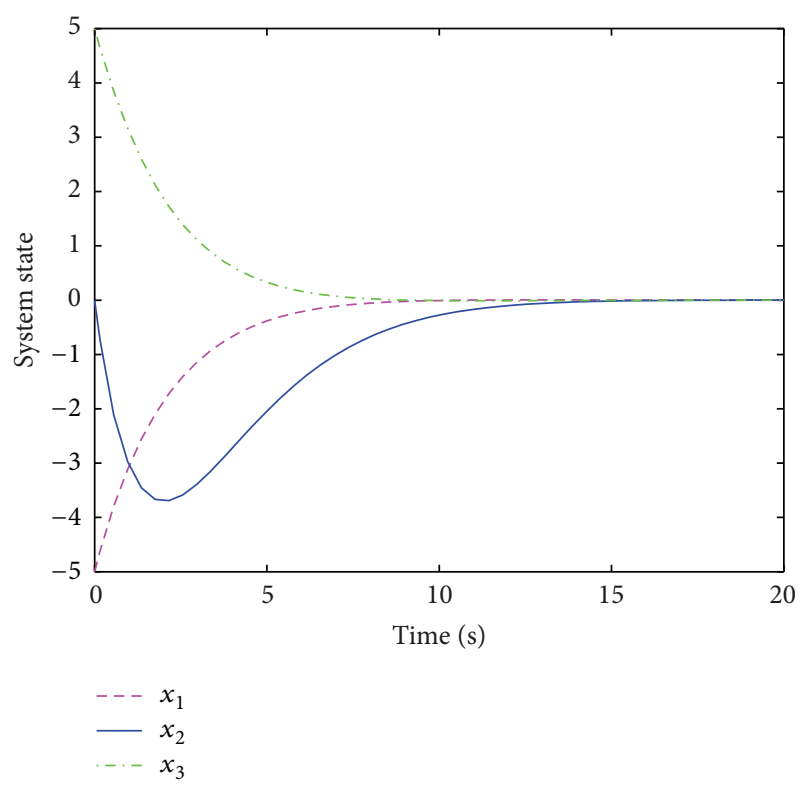

FIGURE 2: State response.

For given $\gamma=1, \alpha=1, \beta=10, R=I, N=5$, according to Theorem 6 , the control matrix is given by

$$
K=Y G^{-1}=\left[\begin{array}{lll}
0.0342 & 0.0155 & -0.9182
\end{array}\right]
$$

To simulate, we take the initial state as $x_{0}=\left[\begin{array}{lll}-5 & 0 & 5\end{array}\right]^{T}$. Figure 2 depicts the trajectory of the system state. The numerical example and simulation demonstrate the effectiveness of the proposed results.

\section{Conclusions}

In this paper, we have considered the finite-time stabilization problems of a class of networked control systems (NCSs) with bounded Markovian packet dropout, based on the iterative approach the NCSs with bounded packet dropout as jump linear systems. The sufficient conditions for finite-time stabilization of the underlying systems are derived via linear matrix inequalities (LMIs) formulation. Lastly, an illustrative example is given to demonstrate the effectiveness of the proposed results.

\section{Conflict of Interests}

The authors declare that there is no conflict of interests regarding the publication of this paper.

\section{Acknowledgment}

This work was partly supported by the University Natural Science Foundation of Anhui Province no. KJ2013A239 and the University Special Foundation for Young Scientists of Anhui Province no. 2013SQRW052ZD.

\section{References}

[1] J. Cao, A. Alofi, A. Al-Mazrooei, and A. Elaiw, "Synchronization of switched interval networks and applications to chaotic neural networks," Abstract and Applied Analysis, vol. 2013, Article ID 940573, 11 pages, 2013.

[2] L. Weiss and E. F. Infante, "Finite time stability under perturbing forces and on product spaces," IEEE Transactions on Automatic Control, vol. 12, pp. 54-59, 1967.

[3] F. Amato and M. Ariola, "Finite-time control of discrete-time linear systems," IEEE Transactions on Automatic Control, vol. 50, no. 5, pp. 724-729, 2005.

[4] F. Amato, M. Ariola, and C. Cosentino, "Finite-time stabilization via dynamic output feedback," Automatica, vol. 42, no. 2, pp. 337-342, 2006.

[5] G. Garcia, S. Tarbouriech, and J. Bernussou, "Finite-time stabilization of linear time-varying continuous systems," IEEE Transactions on Automatic Control, vol. 54, no. 2, pp. 364-369, 2009.

[6] L. Liu and J. Sun, "Finite-time stabilization of linear systems via impulsive control," International Journal of Control, vol. 81, no. 6, pp. 905-909, 2008.

[7] S. Zhao, J. Sun, and L. Liu, "Finite-time stability of linear timevarying singular systems with impulsive effects," International Journal of Control, vol. 81, no. 11, pp. 1824-1829, 2008.

[8] F. Amato, R. Ambrosino, M. Ariola, and C. Cosentino, "Finitetime stability of linear time-varying systems with jumps," Automatica, vol. 45, no. 5, pp. 1354-1358, 2009.

[9] F. Amato, C. Cosentino, and A. Merola, "Sufficient conditions for finite-time stability and stabilization of nonlinear quadratic systems," IEEE Transactions on Automatic Control, vol. 55, no. 2, pp. 430-434, 2010.

[10] F. Amato, M. Ariola, and C. Cosentino, "Finite-time stability of linear time-varying systems: analysis and controller design," IEEE Transactions on Automatic Control, vol. 55, no. 4, pp. 10031008,2010

[11] F. Amato, M. Ariola, and C. Cosentino, "Finite-time control of discrete-time linear systems: analysis and design conditions," Automatica, vol. 46, no. 5, pp. 919-924, 2010.

[12] W. Xiang and J. Xiao, "Finite-time stability and stabilisation for switched linear systems," International Journal of Systems Science, vol. 44, no. 2, pp. 384-400, 2013.

[13] X. Luan, F. Liu, and P. Shi, "Robust finite-time control for a class of extended stochastic switching systems," International Journal of Systems Science, vol. 42, no. 7, pp. 1197-1205, 2011.

[14] D. Yang and K.-Y. Cai, "Finite-time reliable guaranteed cost fuzzy control for discrete-time nonlinear systems," International Journal of Systems Science, vol. 42, no. 1, pp. 121-128, 2011.

[15] X. Yang and J. Cao, "Finite-time stochastic synchronization of complex networks," Applied Mathematical Modelling, vol. 34, no. 11, pp. 3631-3641, 2010.

[16] C. Zheng and J. Cao, "Finite-time synchronization of singular hybrid coupled networks," Journal of Applied Mathematics, vol. 2013, Article ID 378376, 8 pages, 2013.

[17] G. C. Walsh and H. Ye, "Scheduling of networked control systems," IEEE Control Systems Magazine, vol. 21, no. 1, pp. 5765, 2001.

[18] A. H. Tahoun and H.-J. Fang, "Adaptive stabilisation of networked control systems tolerant to unknown actuator failures," International Journal of Systems Science, vol. 42, no. 7, pp. 11551164, 2011. 
[19] M. Liu and J. You, "Observer-based controller design for networked control systems with sensor quantisation and random communication delay," International Journal of Systems Science, vol. 43, no. 10, pp. 1901-1912, 2012.

[20] P. Wang, C. Z. Han, and B. C. Ding, "Stability of discrete-time networked control systems and its extension for robust $H_{\infty}$ control," International Journal of Systems Science, vol. 44, no. 2, pp. 275-288, 2013.

[21] D. Yue, Q. L. Han, and C. Peng, "State feedback controller design of networked control systems," IEEE Transactions on Circuits and Systems II: Express Briefs, vol. 51, no. 11, pp. 640-644, 2004.

[22] L. Zhang, Y. Shi, T. Chen, and B. Huang, "A new method for stabilization of networked control systems with random delays," IEEE Transactions on Automatic Control, vol. 50, no. 8, pp. 11771181, 2005.

[23] J. Y. Yu, L. Wang, and M. Yu, "Stabilization of networked control systems via packet-loss dependent output feedback controllers," in Proceedings of the American Control Conference, pp. 36203625, 2008.

[24] D. Zhang and X. Wang, "Static output feedback control of networked control systems with packet dropout," International Journal of Systems Science, vol. 43, no. 4, pp. 665-672, 2012.

[25] X. M. Zhang, Y. F. Zheng, and G. P. Lu, "Stochastic stability of networked control systems with network-induced delay and data dropout," Journal of Control Theory and Applications, vol. 6, no. 4, pp. 405-409, 2008.

[26] Y. Sun and S. Qin, "Stability analysis and controller design for networked control systems with random time delay," International Journal of Systems Science, vol. 42, no. 3, pp. 359-367, 2011.

[27] D. Yue, Q.-L. Han, and J. Lam, "Network-based robust $H_{\infty}$ control of systems with uncertainty," Automatica, vol. 41, no. 6 , pp. 999-1007, 2005. 


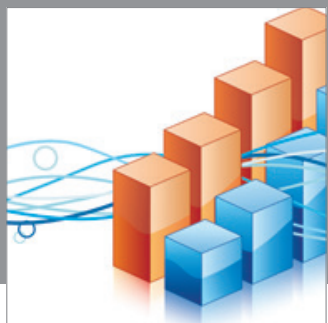

Advances in

Operations Research

mansans

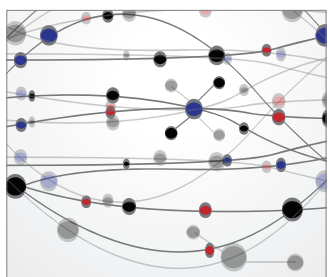

The Scientific World Journal
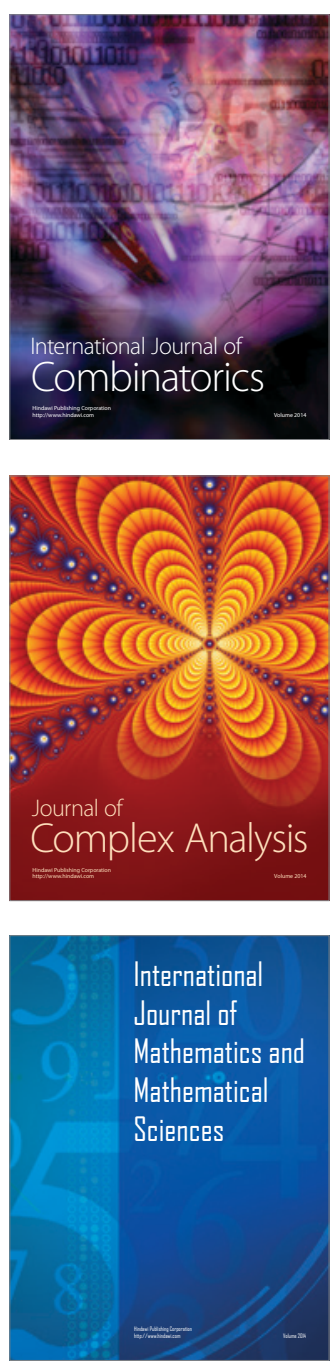
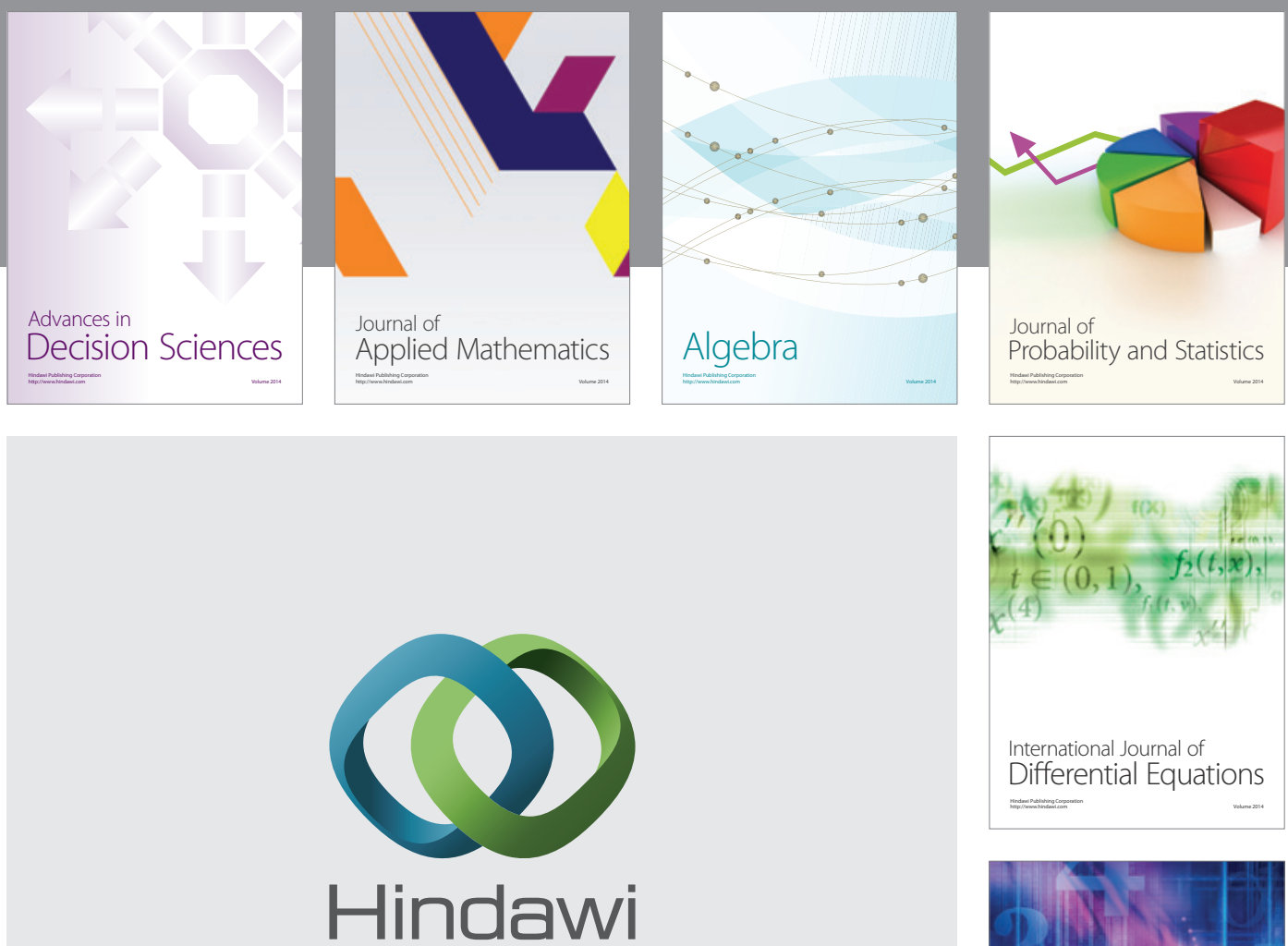

Submit your manuscripts at http://www.hindawi.com
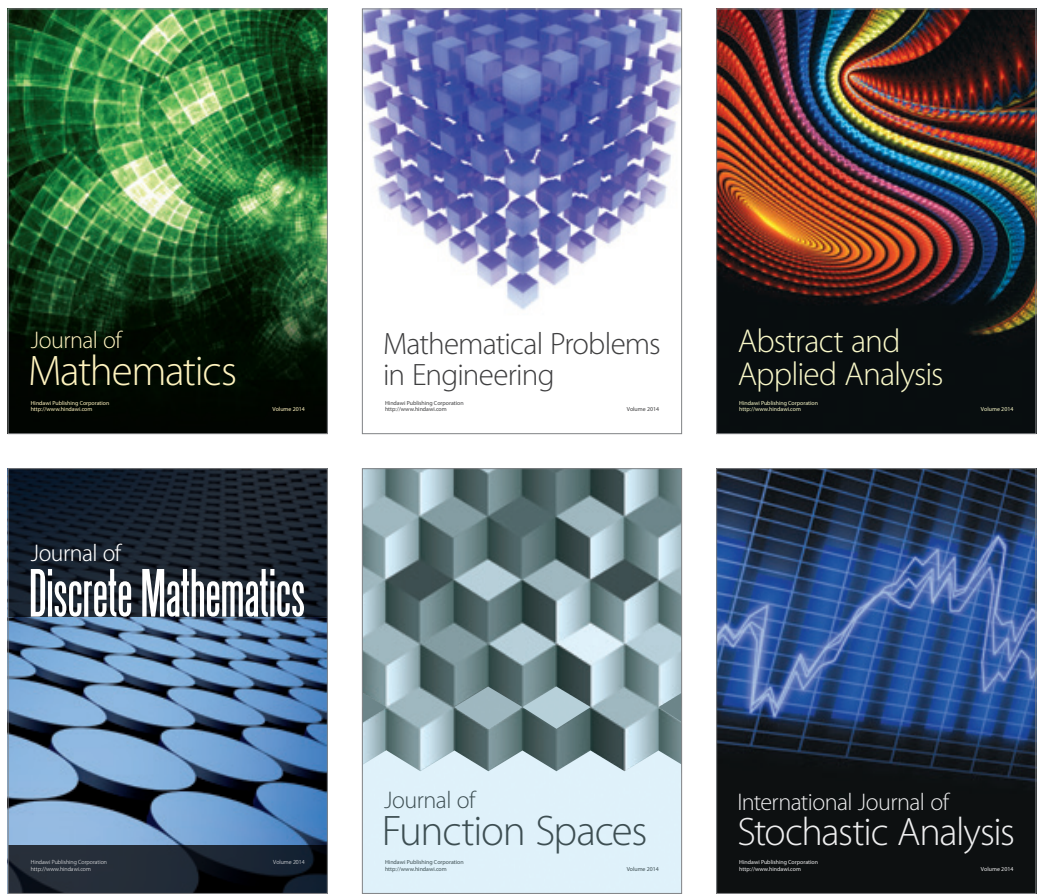

Journal of

Function Spaces

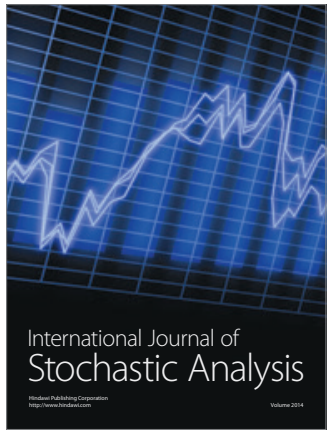

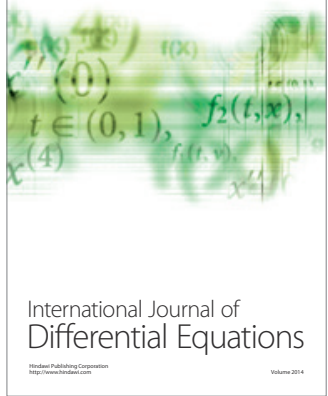
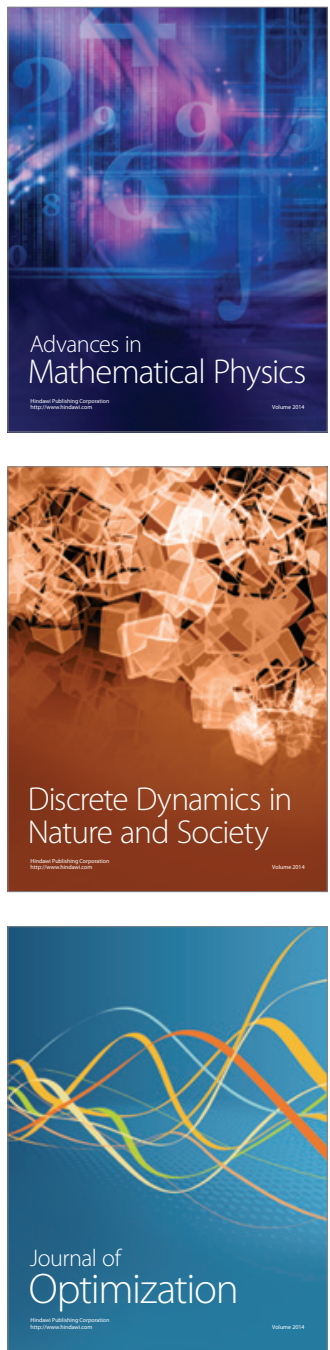